\title{
Positron annihilation spectroscopy in materials science
}

\author{
C S SUNDAR \\ Materials Science Division, Indira Gandhi Centre for Atomic Research, Kalpakkam 603102. \\ India

\begin{abstract}
The application of positron annihilation spectroscopy to the study of defects in materials is illustrated through several examples drawn from our work. These include the study of vacancy clustering in metals, clustering of He atoms to form bubbles, and solute clustering in alloys. Results of studies on novel materials such as quasicrystals, cuprate superconductors and fullerenes are also presented.
\end{abstract}

Keywords. Positron annihilation spectroscopy; clustering; helium bubbles; quasicrystals; cuprate superconductors; fullerenes.

\section{Introduction}

When energetic positrons from a radioactive source enter condensed medium, they rapidly lose their energy by collisions with electrons and ions. After a somewhat longer period, characteristic of the medium, their annihilation is announced by the emergence of energetic photons, whose energy, momenta and time of emission can be measured with great precision with modern detector systems. The utility of positron annihilation studies of condensed matter (for reviews see: West 1973; Brandt and Dupasquier 1983) relies on the fact that, while in principle the characteristics of the annihilation process involve sophisticated considerations of quantum electrodynamics, nevertheless depend almost entirely on the initial state of the positron-many electron system. In perfect crystalline materials, the positron exists in a periodic Bloch state, and from a measurement of the momentum of the annihilation photons, information on the electron momentum distribution can be obtained. This forms the basis of an important area of application of positron annihilation to the investigation of electronic structure (Berko 1983) of materiais. In the presence of defects, in particular vacancy-type defects, positrons are trapped at these sites and the resultant annihilation characteristics can be used to characterize the defect (Nieminen 1983). In the last two decades, these two streams of applications have been refined and several detailed and interesting studies pertaining to electronic structure and defect properties of materials have been carried out. In this article, the application of positron annihilation spectroscopy (PAS) to defects is emphasized with example drawn from our own work.

The format of this paper is as follows: In $\S 2$ a brief introduction to the positron annihilation technique is provided, following which the physical basis for the investigation of a variety of defect-related phenomena are presented in $\S 3$. The application of PAS to the study of clustering of vacancies, helium in metals, and solute clusters is discussed in the subsequent sections. The last decade has seen the discovery of several new classes of materials such as quasicrystals, high-temperature superconductors, fullerenes, etc. In all these materials, PAS has been used with a view to obtain information on structure and electronic properties. Our own efforts in this direction are contained in $\$ 4$. Finally a summary and future outlook are presented. 


\section{Positron annihilation spectroscopy}

The basic aspects of PAS (West 1973) can be best explained with the help of the schematic shown in figure 1. Positrons emitted from a radioactive source such as ${ }^{22} \mathrm{NaCl}$ are injected into the solid, wherein they thermalize and annihilate with one of the electrons in the medium resulting in the emission of two annihilation photons of $0.511 \mathrm{MeV}\left(=m_{o} C^{2}\right)$. The annihilation rate (inverse of lifetime) is determined by the electron density at the site of the positron; in metallic systems the lifetime is $\sim 150$ ps. Positron lifetime measurements are carried out by measuring the time delay between the $1.28 \mathrm{MeV}$ gamma ray which signals the birth of the positron and the $0.511 \mathrm{MeV}$ annihilation photons using ultrafast timing methods derived from nuclear spectroscopy. The annihilation radiation also carries information concerning the momentum of the electron-positron pair. Due to the momentum of the electronpositron pair, which is mainly due to the motion of electrons in the material, there is a slight departure of a few milliradians from collinearity. Thus, from a measurement of the angular correlation of the annihilation radiation, information concerning the electron momentum distribution and Fermi surface can be obtained. Another consequence of the momentum of the annihilating electron-positron pair is the Doppler broadening of the annihilation radiation lineshape which can be measured using a high-energy resolution germanium detector.

\section{Defect spectroscopy with positrons}

In a perfect crystal, the positron moving in the periodic potential of ions and electrons exists in a periodic Bloch state. Due to strong Coulomb repulsion from the positiveion cores, the positron density distribution has a maximum in the interstitial regions

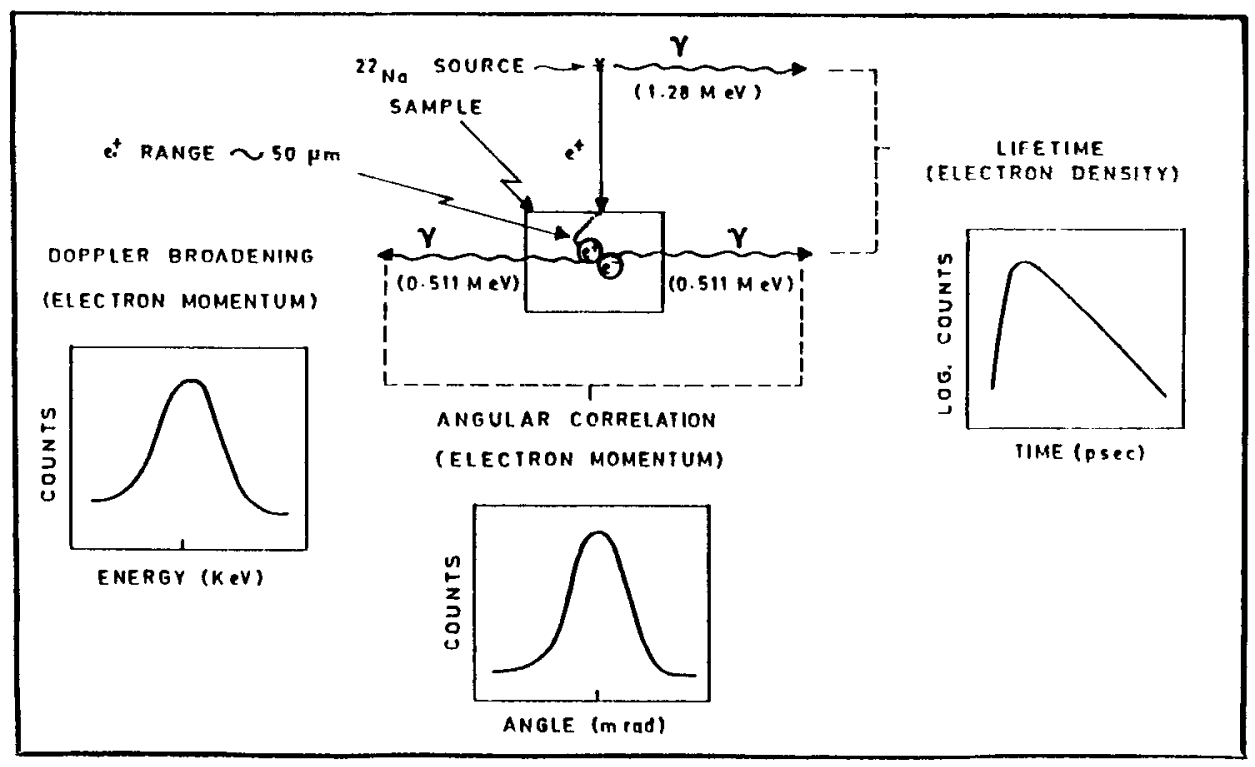

Figure 1. Schematic of positron annihilation spectroscopy. 
and the positron mainly annihilates with the valence electrons with a small contribution from the core electrons. Positron behaviour in crystalline materials is drastically affected in the presence of vacancy-type defects. The absence of ion core results in a strong attractive potential for the positron at which it is trapped (Nieminen 1983). At vacancy-type defects, the total electron density is smaller than in the bulk crystal, which leads to an increase in lifetime. With increase in the concentration of defects (beyond $10^{-7}$ ) an increasing fraction of positrons are trapped at defects, and finally at concentrations greater than $10^{-4}$ all the positrons annihilate from the trapped state. The changes in the annihilation characteristics with defect concentration are described quantitatively in terms of the trapping model (West 1973; Brandt and Dupasquier 1983). From a study of the variation of annihilation characteristics with temperature, the evolution of equilibrium vacancy concentration with temperature can be followed and this forms the basis of one of the most precise methods for the determination of vacancy formation energy.

The annihilation characteristics of the positron trapped at defects is sensitively dependent on the size and even geometry of defect clusters (Nieminen 1983). For example, with increase in the size of the vacancy cluster, the positron gets more localized at the defect and consequently overlaps less with the electron distribution around the defect. This results in an increase in the lifetime with the size of the defect cluster. The association of the experimentally measured lifetime with a specific defect is made possible by developments in the theoretical calculations of positron density distribution and annihilation characteristics at defects (Puska and Nieminen 1983; Bharathi 1988). Briefly, these calculations proceed by numerically solving the Schrödinger equation for the positron with the positron potential given as a superposition of the Hartree electrostatic potential and the electron-positron correlation energy. The annihilation rate is then evaluated from the overlap of the positron density with the enhanced electron density at the site of the positron. Such calculations have now been carried out for a variety of defects such as monovacancies, vacancy clusters, decorated vacancy clusters, solute clusters, etc. Figure 2 shows the calculated values (Amarendra et al 1992) of positron lifetimes as a function of the size of vacancy cluster in $\mathrm{Ni}$. Also shown in the figure is the reduction lifetime with He decoration

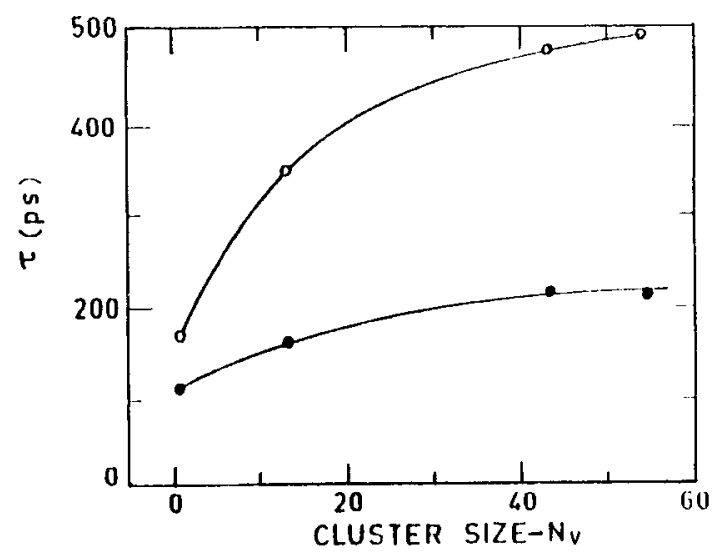

Figure 2. Variation of positron lifetime as a function of cluster size in a pure vacancy cluster ( $O$ ) and at a He-decorated vacancy cluster (O) (From Amarendra et al 1992). 
of the vacancy cluster. The extreme sensitivity of the positron lifetime to the size of small vacancy clusters and the influence of decoration with gas atoms has been exploited in the study of vacancy clustering in metals (Sundar et al 1986) and in the investigation of nucleation and growth of helium bubbles in alpha-irradiated metals (Viswanathan and Amarendra 1990; Gopinathan and Rajaraman 1994). The annihilation characteristics are also seen (see $\S 3.3$ ) to be sensitive to the presence of small solute clusters in quenched alloys, and this has been used (Bharathi 1988) in the study of early stages of phase separation in quenched alloys. An illustrative example of each of these studies is presented in the following.

\subsection{Vacancy clustering in metals}

The evolution of defect structure in cold-worked $\mathrm{Ni}$ with temperature has been monitored (Sundar et al 1986) by positron lifetime measurements as a function of isochronal annealing temperature. The measured lifetime spectra have been analysed in terms of two lifetime components $\tau_{1}$ and $\tau_{2}$, and the intensity of the second component $I_{2}$, and the results are shown in figure 3. In the temperature range up to $160^{\circ} \mathrm{C}$, the lifetime spectrum has a single component of $170 \mathrm{ps}$, which can be associated (c.f. figure 2) with the annihilation at monovacancies and dislocations. In the temperature range $160-400^{\circ} \mathrm{C}$, the lifetime and intensity of the second component increases. The increase in $\tau_{2}$ signals the agglomeration of mobile vacancies resulting in the formation of vacancy clusters (c.f. figure 2). In addition to the above mentioned

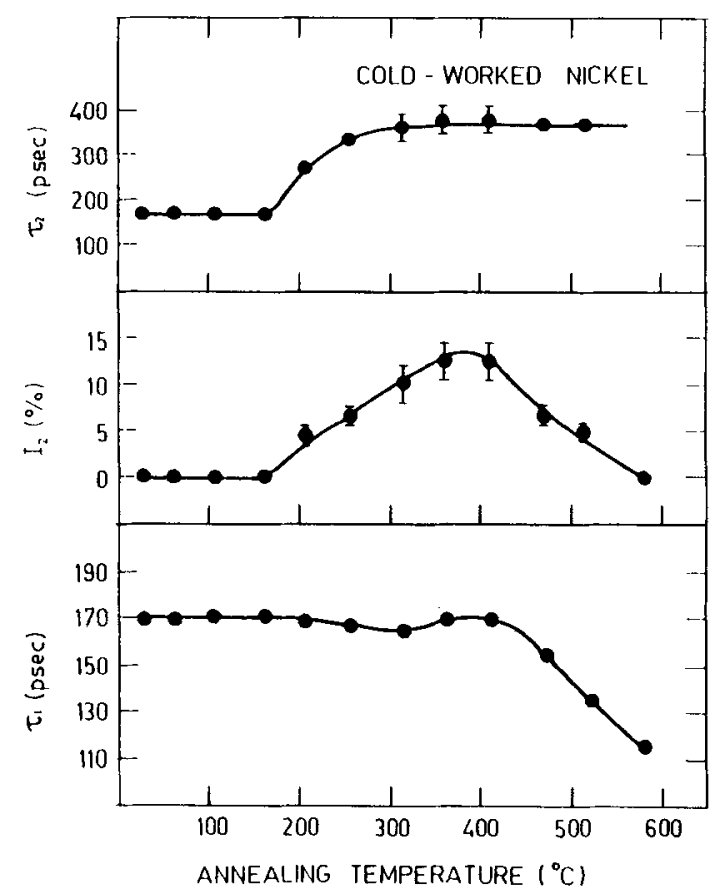

Figure 3. Variation of lifetime parameters as a function of isochronal annealing temperature in cold-worked Ni (From Sundar et al 1986). 
isochronal annealing experiments, through isothermal annealing experiments, the kinetics of clustering of vacancies has been studied. Further, the observed variation of lifetime with annealing temperature and time has been quantitatively understood (Sundar et al 1986) by modelling the evolution of vacancy clusters using chemicalkinetic rate equations coupled with the theoretically calculated values of positron lifetime vs cluster size as shown in figure 2.

The above example illustrates how the results from positron lifetime experiments are used to derive information about the evolution of small vacancy clusters with temperature and time. Extensive studies on the clustering of vacancies during isochronal annealing have been carried out in pure metals and to study the influence of substitutional and interstitial impurities (Kajcsos and Szeles 1992). Several experiments have also been carried out on technologically important materials such as stainless steels (Viswanathan 1989). A recent example of such a study is the investigation of the formation of $\mathrm{TiC}$ precipitates, which play an important role in improving the radiation resistance of stainless steel (Rajaraman et al 1994a).

\subsection{Helium clustering in metals}

The introduction of helium into metals by direct injection or by $(\mathrm{n}, \alpha)$ reactions, occurring during neutron irradiation in a reactor, leads to several deleterious effects such as the nucleation and growth of voids, embrittlement, and blistering of metal surfaces (Donnelly and Evans 1991). The clustering of helium atoms to bubbles proceeds through the formation of $\mathrm{He}_{m}-\mathrm{V}_{n}$ complexes, which act as bubble nuclei, and these grow by the migration and coalescence or Ostwald ripening process. PAS with its sensitivity to small helium-decorated vacancy clusters can be used to follow the evolution of He clusters (Viswanathan and Amarendra 1991; Gopinathan and Rajaraman 1994). This is illustrated by the results of isochronal annealing studies (Amarendra et al 1992) in alpha-irradiated Ni shown in figure 4. In the as-irradiated state, a shorter lifetime of $140 \mathrm{ps}$ with $70 \%$ intensity and a longer lifetime of $240 \mathrm{ps}$ with $30 \%$ intensity are seen. On the basis of theoretical calculations of positron lifetime, the shorter lifetime can be associated with $\mathrm{He}_{4} \mathrm{~V}$ complexes whereas the longer-lifetime component to small vacancy clusters containing about five vacancies. An important feature of the annealing curve shown in figure 4 is that between 500 and $750 \mathrm{~K} \tau_{1}$ decreases sharply towards the bulk value. $\tau_{2}$ decreases exhibiting a minimum, and $I_{2}$ increases to a maximum. These trends in lifetime parameters can be understood (Amarendra et al 1992) as due to helium decoration of vacancy clusters leading to the formation of stable bubble nuclei. The increase in $\tau_{2}$ and the corresponding decrease in $I_{2}$ beyond $750 \mathrm{~K}$ correspond to the growth in size of bubbles with a decreasing concentration. Thus from isochronal annealing experiments, the stages corresponding to the nucleation and growth of helium bubbles can be identified.

The measured positron parameters can be used to obtain the bubble parameters, viz. the concentration $\left(C_{\mathrm{B}}\right)$, radius $\left(r_{\mathrm{B}}\right)$ and helium atom density $\left(n_{\mathrm{He}}\right)$, on the basis of positron surface state model (Jensen and Nieminen 1987). According to this model, the annihilation rate of the positron trapped within the helium bubble is determined both by its overlap with the bubble surface and the helium atom density within the bubble. Further, the fraction of positrons trapped inside the bubbles is dependent 


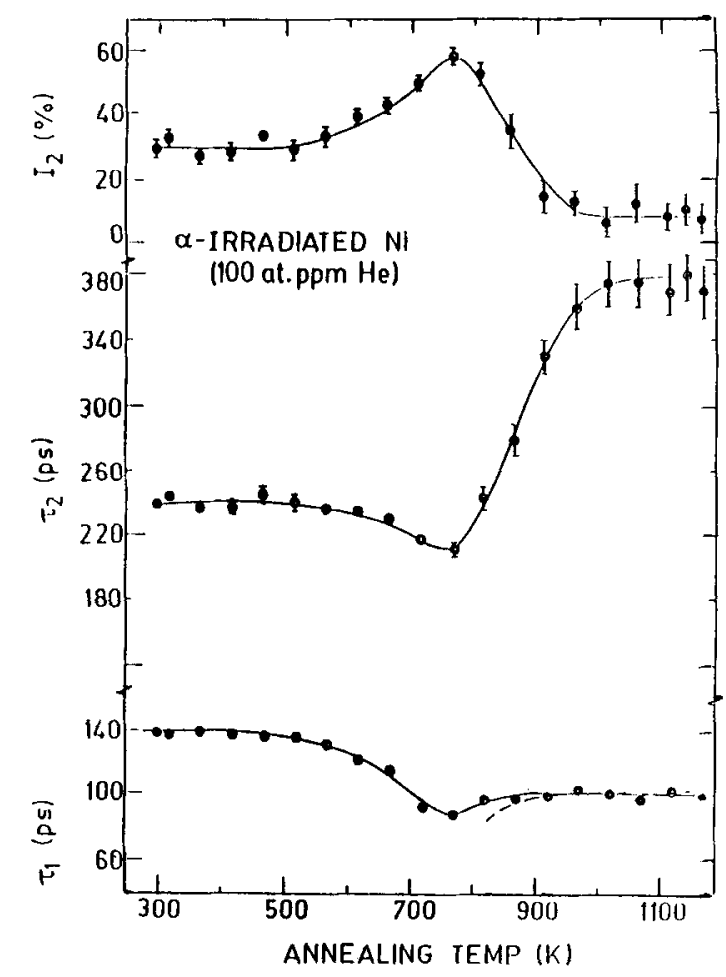

Figure 4. Variation of lifetime parameters as a function of isochronal annealing temperature for $\alpha$-irradiated $\mathrm{Ni}$ (100 at. ppm He). The dashed line indicates $\tau_{1}$ value evaluated using the two-state trapping model (From Amarendra et al 1992).

on both the bubble concentration and radius. This, together with the helium inventory equation, can be used to obtain (Viswanathan and Amarendra 1991) both $C_{\mathrm{B}}$ and $r_{\mathrm{B}}$. The results of such an analysis carried out in the case of $\mathrm{Ni}$ are shown in figure 5. Studies on helium bubble growth have been carried out to investigate the effect of irradiation temperature, dose, the influence of impurities, etc and studies on the kinetics of bubble growth have also been carried out (Amarendra 1990; Amarendra et al 1992; Rajaraman 1993; Rajaraman et al 1994b). In recent experiments on $\alpha$-irradiated Pd (Rajaraman et al 1994b) coupled with theoretical modelling, the influence of helium density on bubble growth kinetics has been established. The detailed studies on the evolution of He clusters has been one of the fruitful applications of PAS.

\subsection{Solute clustering in quenched alloys}

The study of early stages of clustering of solute atoms in alloys resulting in the formation of precipitates has been a topic of considerable interest in physical metallurgy (de Fontaine 1975) and has been extensively investigated by diffraction methods and microscopy. As a result of experimental and theoretical studies carried out in our laboratory (Bharathi 1988; Bharathi and Chakraborty 1988), it has been 
shown that PAS can be profitably used to investigate this phenomenon. The sensitivity of positrons to the formation of solute clusters accrues from the preferential affinity of the positron to one of the constituents of the binary alloy, such as $\mathrm{Ag}$ in $\mathrm{Al}-\mathrm{Ag}$ alloy. Thus, if the $\mathrm{Ag}$ atoms cluster together, then the attractive potential for the positron may become large enough to completely localize the positron at the $\mathrm{Ag}$ cluster. The annihilation characteristics are seen to be sensitive to the size, geometry and composition of the solute cluster in alloys (Bharathi 1988; Bharathi and Sundar $1988,1992)$. Figure 6 shows the sensitive dependence of the lifetime as a function of the size of Ag cluster in Al matrix. The lifetime starting from a value characteristic of pure $\mathrm{Al}$ decreases towards bulk $\mathrm{Ag}$ value as the cluster size increases.

The results of in situ Doppler broadening lineshape measurements in quenched $\mathrm{Al}-1 \% \mathrm{Ag}$ alloy are shown in figure 7 . In the as-quenched alloy the lineshape parameter is close to that in $\mathrm{Ag}$. This can be understood in terms of positron trapping and annihilation from $\mathrm{Ag}$-rich Guinier-Preston (GP) zones. In the temperature range $100-200^{\circ} \mathrm{C}$ the lineshape parameter sharply increases and subsequently merges with
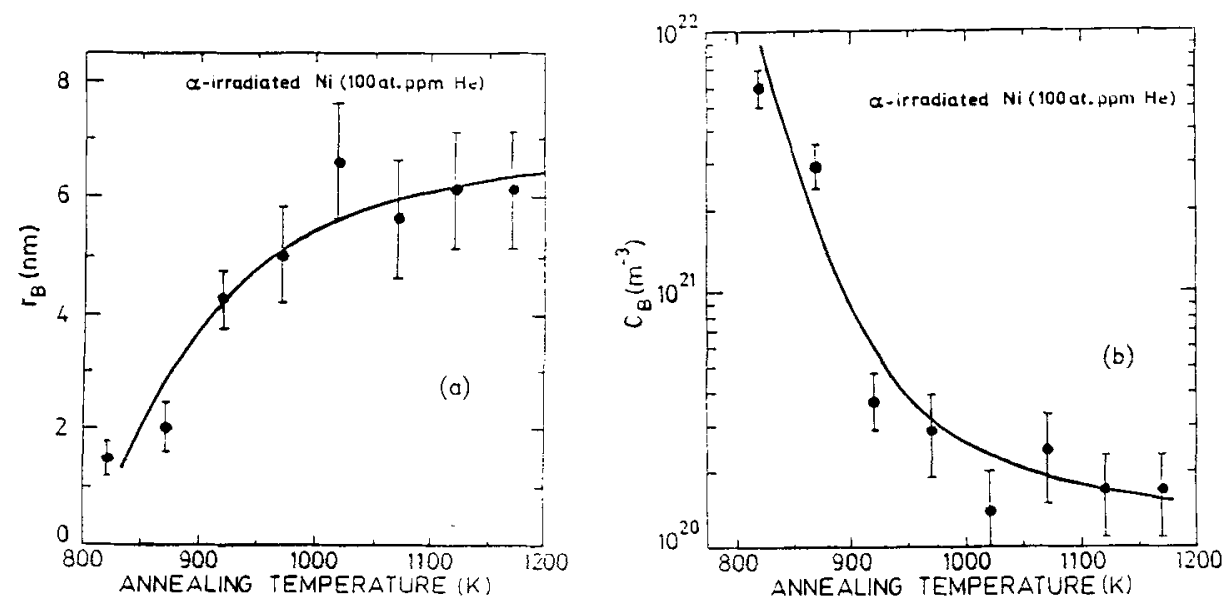

Figure 5. Variation of (a) the He bubble radius $r_{B}$ and (b) bubble concentration $C_{B}$ as a function of annealing temperature as obtained from the analysis of data shown in figure 4.

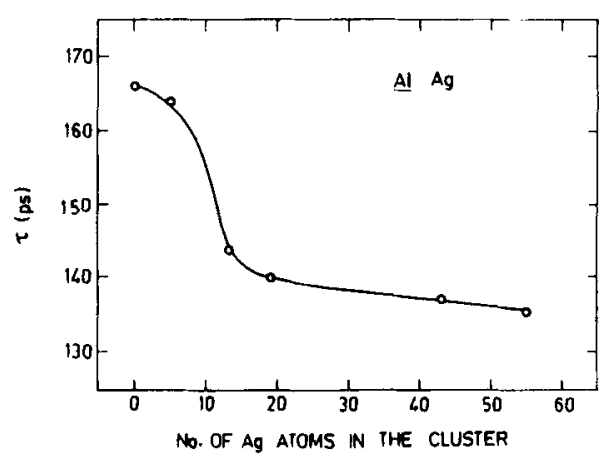

Figure 6. Theoretically calculated variation of positron lifetime as a function the size of Ag cluster in Al matrix (From Bharathi and Sundar 1988). 
results for pure Al. This sharp increase in the $I$ parameter is associated with decrease in $\mathrm{Ag}$ content of GP zones rather than coarsening, leading to reduction in the number density of GP zones. From the measured value of the $I$ parameter, the composition of GP zones at various temperatures can be obtained, and this provides a novel method for determining the GP zone solvus (Bharathi and Sundar 1988). The results obtained from positron experiments are shown in figure 8 . In view of the inherent sensitivity of positrons to vacancies, the role of quenched-in vacancies in the formation

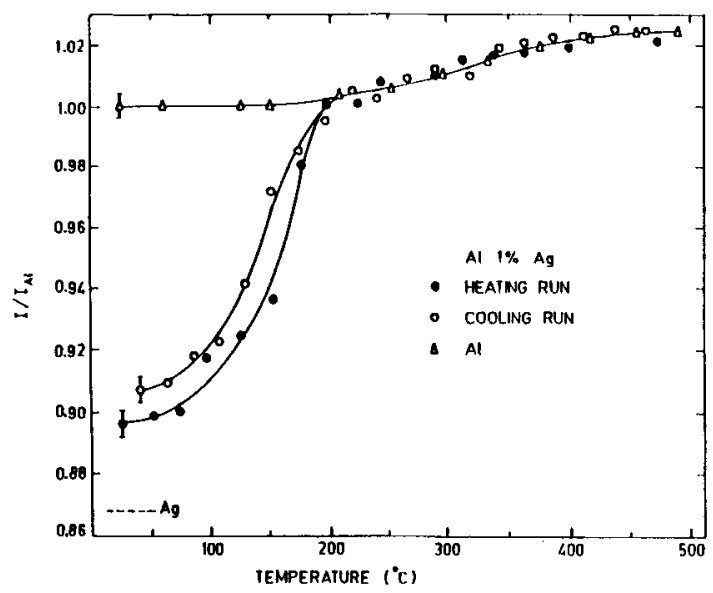

Figure 7. Temperature dependence of Doppler lineshape parameter, with reference to $\mathrm{Al}$, in $\mathrm{Al}-1 \% \mathrm{Ag}$ alloy (From Bharathi and Sundar 1988).

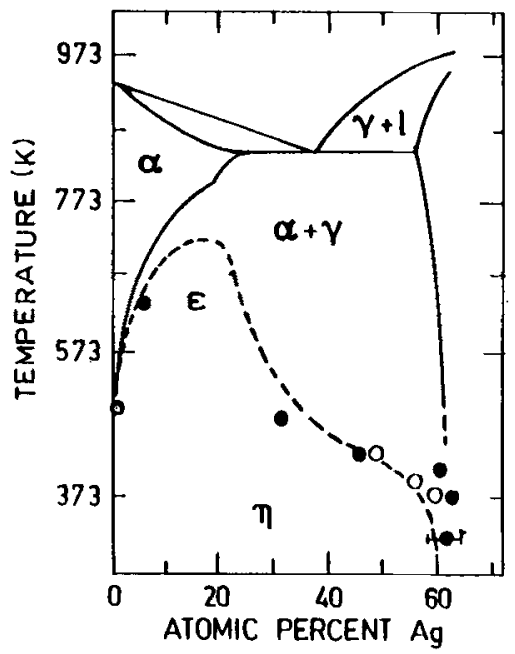

Figure 8. Phase diagram of $\mathrm{Al}-\mathrm{Ag}$ alloy, in which the data obtained from Doppler measurements in $\mathrm{Al}-1 \% \mathrm{Ag}$ (closed circles) and $\mathrm{Al}-6 \% \mathrm{Ag}$ (open circles) alloy are plotted. These data points are obtained by analysis of the lineshape parameter in the alloy in terms of the weighted average of the lineshape parameter in $\mathrm{Al}$ and $\mathrm{Ag}$ (From Bharathi and Sundar 1988). 
of GP zones can be investigated. In addition to the study of the early stages of the clustering process, PAS can also be used to investigate the later stages of precipitation process by probing the defects generated at the interfaces of the precipitate/matrix interface, as has been carried out in $\mathrm{Al}-22 \% \mathrm{Zn}$ alloy (Bharathi et al 1988a).

\section{Studies on novel materials}

Apart from the above examples of using PAS as a tool for detailed studies of defects in crystalline metals, in our laboratory accent has also been on the application of PAS to the investigation of defects and electronic properties of novel materials. Following this approach, several studies have been carried out on metallic glasses (Gopinathan and Sundar 1984), quasicrystals, cuprate superconductors, and, recently, fullerenes. An account of some of the salient results is presented in the following after a brief background of the interest in the particular system.

\subsection{Quasicrystals}

Quasicrystals represent a new class of ordered structures wherein there is long-range translational order and crystallographically forbidden rotational symmetry and there has been a tremendous interest in trying to understand the atomic structure and stability of quasicrystals (DiVincenzo and Steinhardt 1991). In addition to diffraction methods, several experimental techniques which are sensitive to the local structure have been employed and these have played a valuable role in establishing the existence of similar icosahedral clusters, such as the MacKay icosahedron, in both the quasicrystalline phase and crystalline approximants (Goldman and Kelton 1993). It is of interest to know if positrons are trapped at the central vacant site of this cluster and thus provide information about the local structure. A priori, this has been the motivation for positron studies in several quasicrystalline systems, such as Al-Mn, $\mathrm{Al}-\mathrm{Mn}-\mathrm{Si}, \mathrm{Al}-\mathrm{Li}-\mathrm{Cu}$ and $\mathrm{Al}-\mathrm{Cu}-\mathrm{Fe}$. Chidambaram et al (1993) observed the presence of a second lifetime component corresponding to small vacancy clusters, of a few ppm concentration, and this has been used to argue in support of the icosahedral glass model rather than models based on Penrose tiling.

In our experiments (Sundar et al 1991a), in situ measurements of positron lifetime as a function of temperature have been carried out in $\mathrm{Al}-\mathrm{Cu}-\mathrm{Fe}$ and the results are shown in figure 9. The lifetime is seen to show a reversible increase in two steps with a plateau in the range $250-400^{\circ} \mathrm{C}$. From studies on several $\mathrm{Al}-\mathrm{Cu}-\mathrm{Fe}$ systems of different compositions, coupled with $\mathrm{X}$-ray diffraction measurements, it has been shown that this reversible behaviour is due to positron trapping at thermally generated phason disorder. As is well known, the presence of phason disorder (Goldman and Kelton 1993) corresponds to flipping of long and short sequence of the ideal Fibonacci sequence and such a flipping can lead to a clustering of long segments. In the context of positron experiments, a clustering of long segments will lead to a local increase in the open volume regions and can account for the increase in lifetime with temperature. It would be interesting to carry out high-resolution positron-lifetime experiments, coupled with theoretical modelling, that could discriminate between thermal vacancies and phason disorder. This will be of great value in separating the 


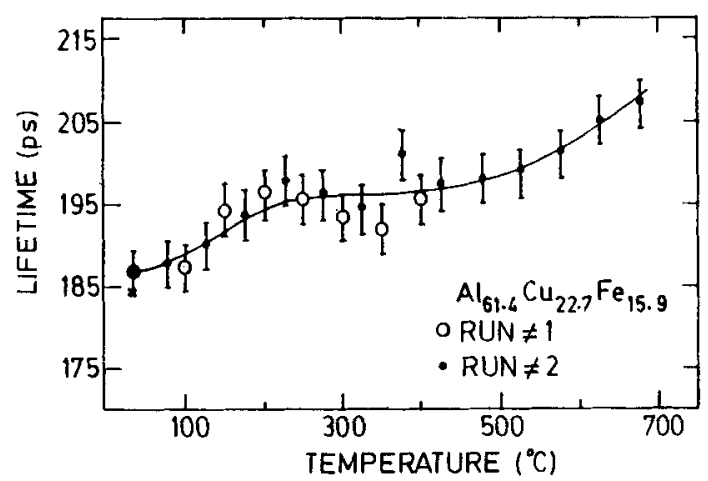

Figure 9. Variation of positron lifetime as a function of sample temperature in quasicrystalline $\mathrm{Al}_{61 \cdot 4} \mathrm{Cu}_{22 \cdot 7} \mathrm{Fe}_{15 \cdot 9}$ (From Sundar et al 1991a).

entropic contributions from vacancies and phason disorder to the stability of quasicrystals (DiVincenzo and Steinhardt 1991).

\subsection{High-temperature superconductors}

The discovery of high-temperature superconductors (HTSC) has initiated enormous activity (Muller and Olsen 1988), both theoretical and experimental, to understand the normal and superconducting states in these systems. PAS has been extensively applied to the study of these materials (for earlier reviews see: Sundar et al 1990a; Sundar and Bharathi 1991) and these studies can be broadly classified into (i) studies on the temperature dependence of annihilation characteristics across $T_{c}$, (ii) studies on structure and defect properties, and (iii) investigation of Fermi surface in these materials.

\subsection{Temperature dependence of positron annihilation characteristics across $T_{\mathrm{c}}$}

The motivation for the studies on the temperature dependence of positron annihilation characteristics has been to see if the annihilation characteristics show any changes across $T_{\mathrm{c}}$ and can provide a clue to the mechanism of superconductivity. The first measurement of the temperature dependence of annihilation characteristics across $T_{\mathrm{c}}$ in $\mathrm{YBa}_{2} \mathrm{Cu}_{3} \mathrm{O}_{7-x}$ (Y 1:2:3) was carried out by Jean et al (1987). In the ceramic superconductor, the positron lifetime and the Doppler lineshape parameter $I$ were observed to decrease below $T_{c}$, whereas no such change was seen in the oxygendeficient nonsuperconducting compound. Since these early measurements, there have been several studies on the Y 1:2:3 system and other cuprate superconductors (for reviews see Sundar et al 1990a). While most of the measurements in Y 1:2:3 indicated a decrease in positron annihilation parameters below $T_{\mathrm{c}}$, in some experiments different kinds of temperature dependences were observed. This has caused considerable confusion in identifying the intrinsic temperature dependence associated with the superconducting transition in HTSC materials. It is plausible that these different temperature dependences arise due to the difference in the disposition of positron 


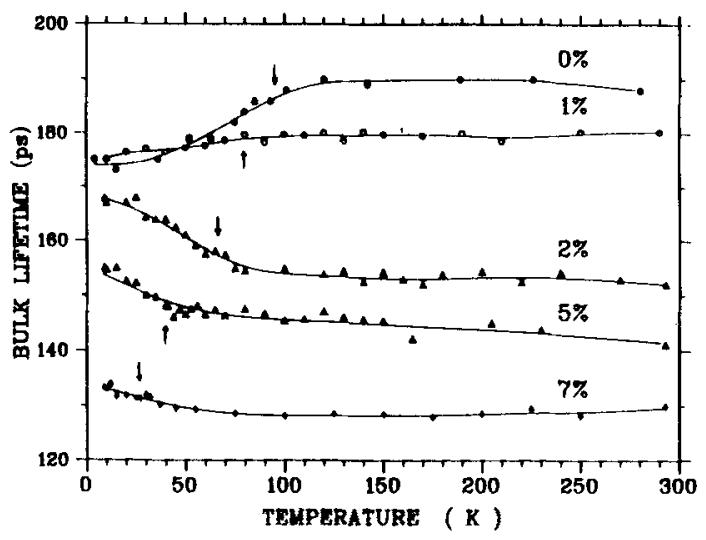

Figure 10. Variation of positron lifetime as a function of temperature in undoped and $\mathrm{Zn}$-doped Y 1:2:3. The arrows are marked at $T_{\mathrm{c}}$ (From Jean et al 1990).

density distribution (PDD) with respect to the superconducting $\mathrm{Cu}-\mathrm{O}$ planes and chains which are the dominant structural features of the Y 1:2:3 structure. In order to check this hypothesis, controlled experiments (Jean et al 1990) on undoped and $\mathrm{Zn}$ - and Ga-doped Y 1:2:3, coupled with theoretical calculations (Bharathi et al 1990) of the PDD, were carried out. Figure 10 shows the results of the experiments on Y 1:2:3 doped with various levels of $\mathrm{Zn}$. It is seen that the decrease in bulk lifetime seen in undoped $Y$ 1:2:3 reverses to an increase in lifetime in $\mathrm{Zn}$-doped $\mathrm{Y}$ $1: 2: 3$. Thus, in the same set of controlled measurement, different temperature dependences were observed, and the variation of lifetime in all cases is seen to be correlated with $T_{\mathrm{c}}$, which decreases with increase in $\mathrm{Zn}$ content.

PDD in Y 1:2:3 has been obtained (Bharathi et al 1990) by solving the Schrödinger equation with the positron potential obtained using the potentials and electron densities from the orthogonalized linear combination of atomic orbitals (OLCAO) band structure calculations. In addition to the calculations of PDD, the electronpositron overlap function, which provides information on the contribution to annihilation from the various atoms in the unit cell, has also been calculated and these results are shown in figure 11. From these, it is seen that in undoped Y $1: 2: 3$, wherein a decrease in lifetime is observed, the PDD is mainly in the region of $\mathrm{Cu}-\mathrm{O}$ chains and the annihilation is dominated by the apical oxygen atom. In the $\mathrm{Zn}$-doped Y 1:2:3, wherein an increase in lifetime is observed, there is a significant positron density in the $\mathrm{Cu}-\mathrm{O}$ planes and the annihilation has large contribution from the planar oxygen atom. With this information, the different temperature dependences, i.e. both a decrease in lifetime as in undoped $\mathrm{Y} 1: 2: 3$ and an increase in lifetime as in $\mathrm{Zn}$-doped Y 1:2:3, can be consistently explained (Bharathi et al 1990; Jean et al 1990 ) in terms of an electron transfer from the planar $O$ atom to the apical $O$ atom. This is schematically shown in figure 12 . An electron transfer from the planar to apical $O$ atom will lead to a decrease in lifetime in undoped $Y 1: 2: 3$ since the annihilation is dominated by the apical $\mathrm{O}$ atom. A similar transfer of electrons will in the case of $\mathrm{Zn}$-doped Y 1:2:3 contribute to an increase in lifetime since there is significant annihilation from the $\mathrm{O}$ atoms of the $\mathrm{Cu}-\mathrm{O}$ layer. 


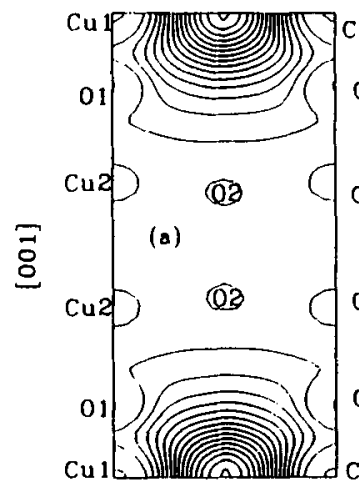

[100]

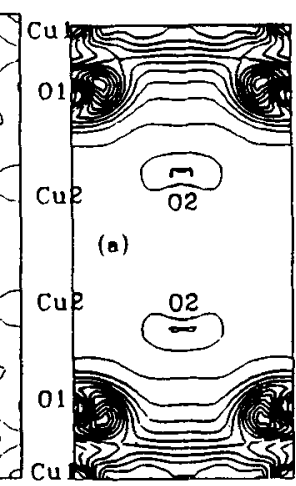

[100]

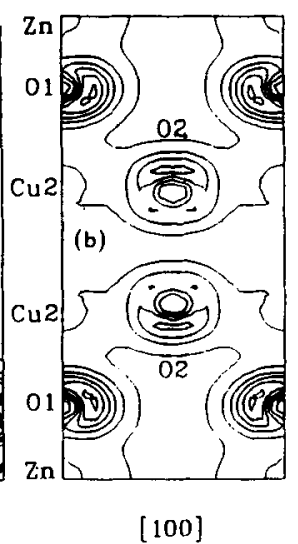

[100]

Figure 11. (a) Contour plot of the positron density distribution in the (010) plane of undoped Y $1: 2: 3$. The maximum in the density is between the $\mathrm{Cu}(1)$ atoms and the contour spacing is $0.005 \mathrm{e}^{+} / \mathrm{a} . \mathrm{u}^{3}$. (b) Contour plot of the electron-positron overlap in the $(010)$ plane of undoped $Y$ 1:2:3, showing the maximum at the apical oxygen atom, $O(1)$. (c) Overlap function in the (010) plane of $\mathrm{Zn}$-doped $\mathrm{Y} 1: 2: 3$, indicating contributions from both the apical and planar oxygen atom $\mathrm{O}(2)$. The contour spacing is $0.05 \mathrm{~ns}^{-1} / \mathrm{a} . \mathrm{u}^{3}$ (From Bharathi et al 1990).

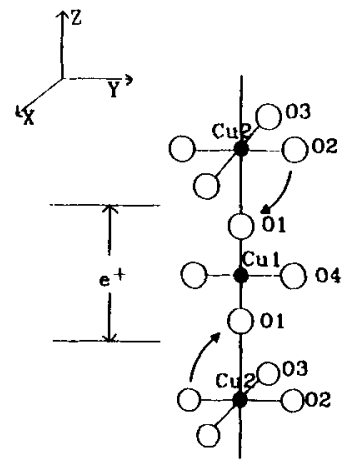

(a)

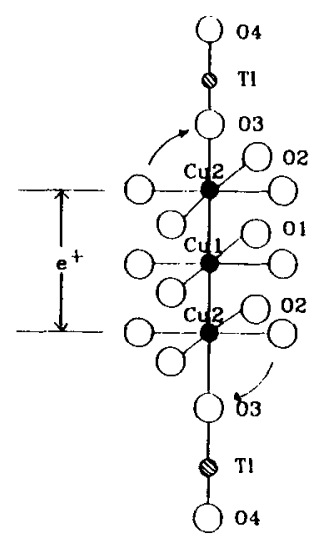

(b)

Figure 12. Schematic diagram of the local electron transfer from the planar to apical oxygen atom in (a) Y 1:2:3 and (b) T] 2:2:2:3. The band shows the range of PDD (From Bharathi et al 1990).

Experiments on the temperature dependence of lifetime across $T_{\mathrm{c}}$ have also been carried out in other cuprate superconductors: $\mathrm{YBa}_{2} \mathrm{Cu}_{4} \mathrm{O}_{8}$ (Sundar et al 1990b), $\mathrm{La}-\mathrm{Sr}-\mathrm{Cu}-\mathrm{O}$ (Jean et al 1988), $\mathrm{Tl}-\mathrm{Ba}-\mathrm{Ca}-\mathrm{Cu}-\mathrm{O}$ (Sundar et al 1990c) and $\mathrm{Bi}-$ $\mathrm{Sr}-\mathrm{Ca}-\mathrm{Cu}-\mathrm{O}$ (Sundar et al 1991c). The variety of temperature dependences of lifetime seen in the various cuprates can be rationalized when the results are analysed in terms of PDD and the electron-positron overlap function. These calculations show 
that the positron's sensitivity to superconductivity arises primarily from the ability to probe the $\mathrm{Cu}-\mathrm{O}$ network in the $\mathrm{Cu}-\mathrm{O}$ layer. The different temperature dependences of lifetime, i.e. both the increase and decrease. can be understood in terms of a model of local electron transfer from the planar oxygen atom to the apical oxygen atom, after taking into account the correct PDD within the unit cell of the cuprate superconductor. It may be remarked that the notion of charge transfer, as used to interpret the positron annihilation data, has also been used to explain several other experimental results in HTSC and also forms the basis of several theoretical models of superconductivity (Bishop et al 1989).

\subsection{Defects and structural studies}

It is well known that in HTSC materials, the defects and in particular the oxygen vacancies play an important role in controlling the structure and superconducting properties (Jorgensen 1991). On quenching the superconducting orthorhombic Y $1: 2: 3$ from elevated temperatures, the oxygen atoms are known to be depleted from the basal plane, containing the $\mathrm{Cu}-\mathrm{O}$ chains, resulting in the formation of nonsuperconducting tetragonal phase. In the $\mathrm{Y} 1: 2: 3$ system, the positron density is in the region of the $\mathrm{Cu}-\mathrm{O}$ chains (c.f. figure 11a) and the annihilation characteristics are sensitive to the oxygen stoichiometry and the ordering of $\mathrm{O}$ atoms in the basal plane (Bharathi et al 1988c). This has been used in the study of the orthorhombic-totetragonal structural transition occurring with increase of quench temperature (Bharathi et al 1988b; Sundar and Bharathi 1991), and the results are shown in figure 13. Also shown in this figure are the results for $\mathrm{NdBa}_{2} \mathrm{Cu}_{3} \mathrm{O}_{7-x}$ (Sundar et al 1991b). The lower orthorhombic-to-tetragonal structural transition temperature in this system can be understood in terms of weakening of the $\mathrm{O}-\mathrm{O}$ interaction in the basal plane arising due to the larger lattice parameters of the $\mathrm{Nd}-\mathrm{Ba}-\mathrm{Cu}-\mathrm{O}$ system compared to Y $1: 2: 3$.

The sensitivity of positron annihilation characteristics to the various ordered phases of $Y 1: 2: 3$ has been used in the study of the decomposition of off-stoichiometric Y 1:2:3, occurring on ageing within the two-phase region (Vasumathi et al 1990).

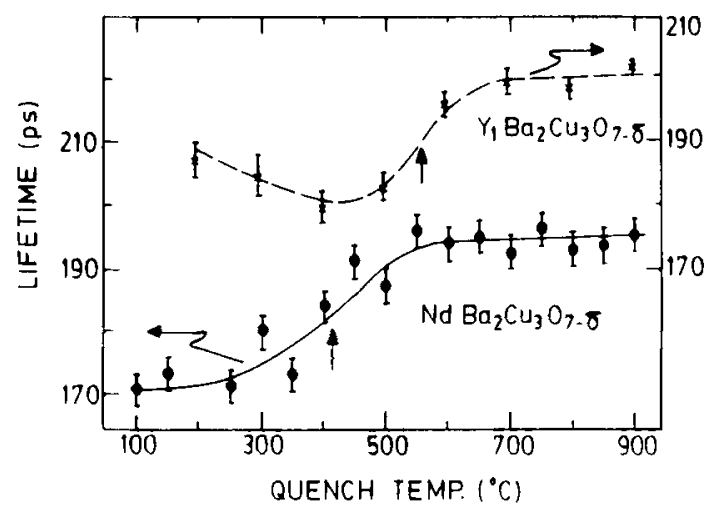

Figure 13. Variation of lifetime as a function of quench temperature in $\mathrm{YBa}_{2} \mathrm{Cu}_{3} \mathrm{O}_{7-x}$ and $\mathrm{NdBa}_{2} \mathrm{Cu}_{3} \mathrm{O}_{7-x}$ (From Sundar et al 1991b). 
Apart from the study of oxygen defects in $\mathrm{Y} 1: 2: 3$, the intercalation of oxygen in $\mathrm{Bi}-\mathrm{Sr}-\mathrm{Ca}-\mathrm{Cu}-\mathrm{O}$ (Sundar et al 1991c) and vacancy defects in $\mathrm{La}-\mathrm{Sr}-\mathrm{Cu}-\mathrm{O}$ (Sundar et al 1992) have also been investigated. The nature of flux-pinning defects that lead to an increase in critical current density on neutron and heavy-ion irradiation is a topic of considerable interest. The increase in critical current density with neutron dose has been correlated ( $\mathrm{Lu}$ et al 1992) with the microvoid density as obtained by positron lifetime measurements. With the increase in realization of the importance of defects in controlling the structure and superconducting properties of HTSC materials (Jorgensen 1991), it is clear that PAS will continue to play an important role.

\subsection{Fermi surface studies}

One of the key issues which has a direct bearing on several theoretical models of high-temperature superconductivity has been the question of the existence of Fermi surface (Pickett et al 1992). Measurement of two-dimensional angular correlation of annihilation radiations (2D-ACAR), which provides information on the electron momentum distribution in solids, has been successfully applied (Berko 1983) to obtain detailed information on the Fermi surface in the A-15 superconductors. With this background, several 2D-ACAR studies have been carried out mainly on the Y $1: 2: 3$ system (Smedskjaer and Bansil 1992; West 1992). An unequivocal answer to the question of the existence of Fermi surface was not obtained for long due to the difficulties associated with the preparation of good-quality single crystals free from defects. Recently, experiments (Haghighi et al 1991) on untwinned crystals have at last provided, definitive evidence for the existence of Fermi surface in good agreement with the LDA predictions (Pickett et al 1992). These experiments coupled with angle-resolved photoemission experiments have helped to clarify the much debated controversy with respect to the description of electronic structure of cuprates.

\subsection{Fullerenes}

During the last three years fullerenes, the close-caged carbon clusters $\mathrm{C}_{60}$ and $\mathrm{C}_{70}$, have attracted considerable attention, both due to their elegant molecular symmetry and the intriguing structural, dynamic and electronic properties of their solid phase (Heiney 1992). One dominant interest in these molecular solids has been in the structural transformations with temperature and pressure which are related to the orientational correlations amongst the molecules. From the point of view of use of PAS in the study of $\mathrm{C}_{60}$, it is firstly of interest to know if the positron probes the interior of the hollow cage structures or the annihilation is from the interstitial regions of the solid. From measurements (Jean et al 1992) of positron lifetime in $\mathrm{C}_{60}$ as a function of pressure (see figure 14), coupled with theoretical calculations of positron density distribution (see figure 15), it has been established that the positron probes the interstitial regions. Interesting effects of the positron dynamics between the interstices to the region within the cage structure have been seen in recent experiments (Sundar et al 1994) of positron lifetime vs temperature. Further, since the positron probes the interstitial region of $\mathrm{C}_{60}$ solid, the annihilation characteristics are sensitive to the effects of intercalation of K (Lou et al 1992). Interesting experiments 


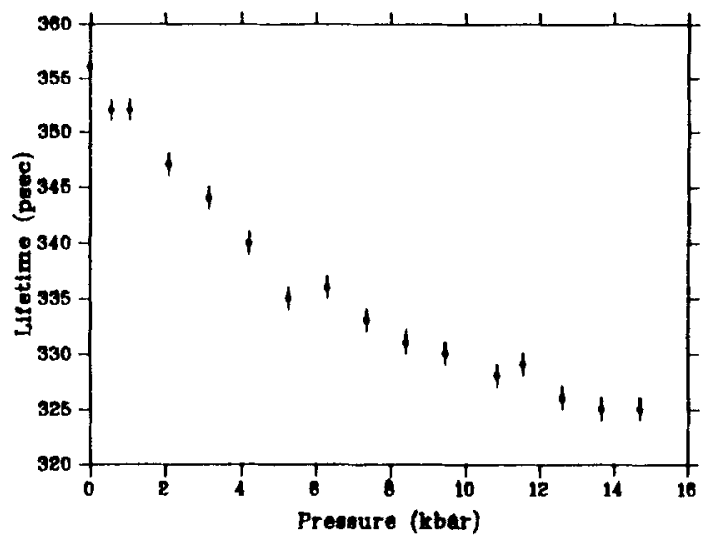

Figure 14. Positron lifetime as a function of pressure in $C_{60}$ (From Jean et al 1992).

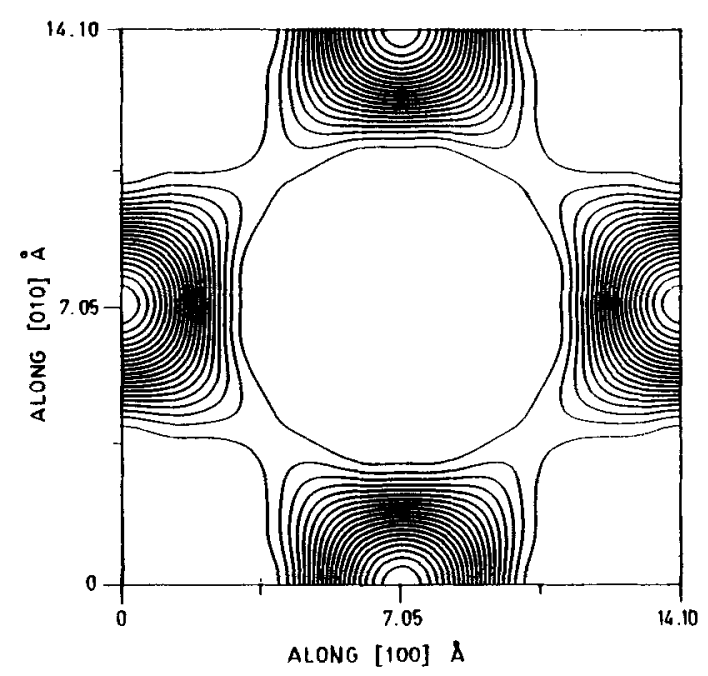

Figure 15. Positron density distribution in the basal plane of $\mathrm{C}_{60}$ showing the maxima in the interstitial region of the fcc lattice (From Sundar et al 1994).

on the decomposition of the off-stoichiometric K-doped phases, and experiments across $T_{\mathfrak{c}}$, are possible, and still remain to be done.

\section{Summary and future outlook}

As a result of extensive work in the last two decades, PAS is now well established as a technique for the investigation of defects in materials, in particular vacancytype defects and small vacancy clusters. With high-resolution positron lifetime measurements, coupled with theoretical calculations, it is now possible to obtain a detailed characterization of defects. The accent in this paper has been to bring out how information related to defects is obtained in positron experiments. Further 
developments will rely on the judicious application of this method to investigate interesting defect-related phenomena in materials science.

The last decade has also seen tremendous progress with respect to the developments in the technique per se. Of particular interest from the point of view of study of defects is the development of low-energy positron beam spectrometers (Mills 1983; Schultz and Lynn 1988). In contrast to the use of continuous distribution of positrons from a radiactive source, in a low-energy positron beam spectrometer, thermalized positrons emitted by the moderator such as W(110) are extracted, magnetically or electrostatically filtered, and accelerated to the few $\mathrm{keV}$ before impinging on the target. The use of monoenergetic positrons of tunable energy provides a method for investigation of defects in the near-surface region, at interfaces, and for depth profiling of defects (Schultz and Lynn 1988). An effort at development of a low energy positron beam spectrometer is being carried out in our laboratory (Viswanathan and Amarendra 1990). Recently, it has also become possible to obtain intense microbeams of positrons which have potential for development of positron microprobe and even positron microscopes. With these developments and an increasing realization that defects play an important role in influencing physical properties, positron studies will continue to play a crucial role in characterization of the defect properties of materials.

\section{Acknowledgements}

I am grateful to all the members of the metal physics laboratory for collaboration on various experiments and discussions of the results presented in this paper: Dr A Bharathi for association with all the experiments and theoretical analysis; Dr K P Gopinathan for initiating me to positron annihilation spectroscopy; and my colleagues Dr G Amarendra, Dr B Viswanathan, Dr Y Hariharan, Smt D Vasumathi, Smt Padma Gopalan, Smt M Premila and Shri R Rajaraman, Shri C M Chittaranjan and Shri J P Jayapandian for collaboration on various experiments and discussions. A part of the work on high-temperature superconductors was carried out while I was a postdoctoral student and I am thankful to Prof. Y C Jean, UMKC, and Prof. C W Chu, University of Houston. Finally, I would like to thank the Materials Research Society of India for giving me an opportunity to present this paper.

\section{References}

Amarendra G 1990 Positron annihilation studies of helium in metals and alloys, $\mathrm{Ph} \mathrm{D}$ thesis, University of Madras, Madras (unpublished)

Amarendra G, Viswanathan B, Bharathi A and Gopinathan K P 1992 Phys. Rev. B45 10231

Berko S 1983 in Positron solid state physics (eds.) W Brandt and A Dupasquier (Amsterdam: North-Holland) p. 64

Bharathi A 1988 Positron annihilation studies of precipitation in aluminium alloys, $\mathrm{Ph} \mathrm{D}$ thesis, University of Madras, Madras (unpublished)

Bharathi A and Chakraborty B 1988 J. Phys. F, 18363

Bharathi A and Sundar C S 1988 in Positron annihilation (eds.) L Dorikens-Vanpraet, M Dorikens and D Segers (Singapore: World Scientific) p. 479

Bharathi A. Sundar C S and Gopinathan K P 1988a Philos. Mag. 58705

Bharathi A, Hariharan Y, Sood A K, Sankara Sastry V. Janawadkar M P and Sundar C S 1988b Europhys. Lett. 6369 
Bharathi A, Sundar C S and Hariharan Y 1988c J. Phys.: Condensed Matter 11467

Bharathi A, Sundar C S, Ching W Y, Jean Y C, Hor P H, Xue Y Y and Chu C W 1990 Phys. Rev. B42 10199

Bharathi A and Sundar C S 1992 Mater. Sci. Forum 105-110 905

Bishop A R, Martin R K, Muller K A and Tesanovic Z 1989 Z. Phys. B76 17

Brandt W and Dupasquier A (eds.) 1983 Positron solid state physics (Amsterdam: North-Holland)

Chidambaram R, Sanyal M K, Raghunathan V S, Nambissan P M G and Sen P 1993 Phys. Rev. B48 3030

de Fontaine D 1975 in Treatise in solid state chemistry (ed.) N B Hannay (New York: Plenum) vol. 5, p. 129

DiVincenzo D P and Steinhardt P J (eds.) 1991 Quasicrystals, The state of art (Singapore: World Scientific)

Donnelly S E and Evans J H (eds.) 1991 Proceedings of the NATO Advanced Workshop on Fundamental Aspects of Inert Gases in Solids (New York: Plenum)

Goldman A I and Kelton R F 1993 Rev. Mod. Phys. 65213

Gopinathan K P and Sundar C S 1984 in Metallic glasses, production, properties and applications (ed.) T R Anantharaman (Switzerland: Trans Tech) p. 115

Gopinathan K P and Rajaraman R 1994 in l0th International Conference on Positron Annihilation, Beijing (in print)

Haghighi H, Kaiser J H, Rayner S, West R N, Liu J Z, Shelton R, Howell R H, Solal F and Fluss M J 1991 Phys. Rev. Lett. 67382

Heiney P E 1992 Phys. Chem. Solids 531333

Jean Y C, Wang S J, Nakanishi H, Hardy W N, Hayden M Y, Kiefl R F, Meng R L, Hor P H, Huang Z J and Chu C W 1987 Phys. Rev. B36 3994

Jean Y C, Kyle J, Nakanishi H, Turchi P E A, Howell R H, Wachs A L, Fluss M J, Meng R L, Hor P H, Huang Z J and Chu C W 1988 Phys. Rev. Lett. 601069

Jean Y C, Sundar C S, Bharathi A, Kyle J, Nakanishi H, Tseng P K, Hor P H, Meng R L, Huang Z J, Chu C W, Wang Z Z, Turchi P E A, Howell R H, Wachs A L and Fluss M J 1990 Phys. Rev. Lett. 641593

Jean Y C, Lu X. Lou Y, Bharathi A, Sundar C S, Lyu Y, Hor P H and Chu C W 1992 Phys. Rev. B45 12126

Jensen K O and Nieminen R M 1987 Phys. Rev. B36 8219

Jorgensen J D 1991 Physics Today June 1991 p. 34

Kajcsos Zs and Szeles Cs (eds) 1992 Positron annihilation (Switzerland: Trans Tech)

Lou Y, Lu X, Dai G H, Ching W Y, Xu Y N, Huang M Z, Tseng P K, Jean Y C, Meng R L, Hor P H and Chu C W 1992 Phys. Rev. B46 2644

Lu X, Wang S J, Sundar C S, Bharathi A, Lyu Y, Ching W Y and Jean Y C 1992 Mater. Sci. Forum $105-110755$

Mills A P Jr 1983 in Positron solid state physics (eds.). W Brandt and A Dupasquier (Amsterdam: North-Holland) p. 432

Muller $\mathrm{J}$ and Olsen $\mathrm{J}$ L (eds.) 1988 High temperature superconductors and materials and mechanisms of superconductivity (Amsterdam: North-Holland)

Nieminen R M 1983 in Positron solid state physics (eds.) W Brandt and A Dupasquier (Amsterdam: North-Holland) p. 359

Pickett W E, Krauker H, Cohen R H and Singh D J 1992 Science 25546

Puska M J and Nieminen R M 1983 J. Phys. F 13333

Rajaraman R 1993 Positron annihilation studies of light impurities in metals, Ph D thesis, University of Madras. Madras (unpublished)

Rajaraman R, Padma Gopalan, Viswanathan B and Venkadesan S 1994a J. Nucl, Mater. (in print)

Rajaraman R, Viswanathan B, Valsakumar M C and Gopinathan K P 1994b Phys. Rev. B50 (in print)

Schultz P J and Lynn K G 1988 Rev. Mod. Phys. 60701

Smedskjaer L C and Bansil A 1992 J. Phys. Chem. Solids 531657

Sundar C S, Bharathi A and Gopinathan K P 1986 in Current trends in the physics of materials (eds.) M Youssouff (Singapore: World Scientific) p. 97

Sundar C S, Bharathi A. Hao L, Jean Y C, Hor P H, Meng R L, Huang Z J and Chu C W 1990a in Superconductivity and its applications (ed.) H S Kwok (New York: Plenum) p. 335

Sundar C S, Bharathi A, Jean Y C, Hor P H, Meng R L, Huang Z J and Chu C W 1990b Phys. Rev. B41 11685

Sundar C S, Bharathi A. Ching W Y, Jean Y C, Hor P H, Meng R L, Huang Z J and Chu C W 1990c Phys. Rev. B42 2193

Sundar C S and Bharathi A 1991 Proc. DAE Solid State Physics Symposium 32A-33A 163

Sundar C S, Chittaranjan C M, Bharathi A, Gopinathan K P and Raghunathan V S 1991a Proc. DAE Solid State Physics Symposium 34C 48 
Sundar C S, Bharathi A, Vasumathi D and Hariharan Y 1991b Proc. DAE Solid State Physics Symposium 33C 176

Sundar C S, Bharathi A, Ching W Y, Jean Y C, Hor P H, Meng R L, Huang Z J and Chu C W 1991c Phys. Rev. B43 13019

Sundar C S, Bharathi A, Vasumathi D and Hariharan Y 1992 Mater. Sci. Forum 105-110 1253

Sundar C S, Bharathi A, Premila M, Padma Gopalan and Hariharan Y 1994 (to be published)

Vasumathi D, Sundar C S, Bharathi A, Sood A K and Hariharan Y 1990 Physica C167 149

Viswanathan B $1989 \mathrm{~J}$. Phys. Condensed Matter 1 SA71

Viswanathan B and Amarendra G 1990 in Positron annihilation and compton scattering (eds.) B K Sharma, P C Jain and R M Singru (New Delhi: Omega Scientific Publishers) p. 29

Viswanathan B and Amarendra G 1991 in Fundamental aspects of inert gases in solids (eds.) S E Donnelly and J H Evans (New York: Plenum) p. 209

West R N 1973 Adv. Phys. 22263

West R N 1992 J. Phys. Chem. Solids 531669 